\title{
Twin removal in digital holography using diffuse illumination
}

\author{
David S. Monaghan, Damien P. Kelly, Nitesh Pandey, and Bryan M. Hennelly* \\ Department of Computer Science, National University of Ireland, Maynooth, County Kildare, Ireland \\ *Corresponding author: bryanh@Cs.nuim.ie
}

Received June 10, 2009; revised October 15, 2009; accepted October 23, 2009;

posted October 29, 2009 (Doc. ID 112665); published November 18, 2009

\begin{abstract}
A method to numerically remove the twin image for inline digital holography, using multiple digital holograms, is discussed. Each individual hologram is recorded by using a statistically independent speckle field to illuminate the object. If the holograms are recorded in this manner and then numerically reconstructed, the twin image appears as a different speckle pattern in each of the reconstructions. By performing specklereduction techniques the presence of the twin image can be greatly reduced. A theoretical model is developed, and experimental results are presented that validate this approach. We show experimentally that the dc object intensity term can also be removed by using this technique. (C) 2009 Optical Society of America

OCIS codes: $090.1995,000.4430$.
\end{abstract}

Holography [1-4] is a technique for capturing the complex amplitude of an optical field, $u_{\mathrm{z}}(x)$. A feature of this interferometric recording process is that three other terms (two dc terms and the so-called twin image), in addition to $u_{\mathrm{z}}(x)$, are also generated. With an in-line setup these terms overlap in space, significantly reducing the quality of the reconstructed hologram [1,3]. Using CCD cameras, it is possible to record holograms digitally and reconstruct them numerically in a computer [5-7]. However, one significant drawback is the limited image detail in such holograms resulting from the finite space-bandwidth product of the CCD camera [8]. Using an off-axis reference field [4] to remove the dc and twin terms further reduces the effective space-bandwidth product. To make best use of the detector resolution in digital holography (DH) it would be optimal to use an inline configuration; however, the dc and twin image terms remain problematic. Phase-shifting interferometric (PSI) techniques can be used to remove these extraneous terms [9-11]; however, several separate captures are required, and thus a temporal aspect is introduced to the capture process; see $[10,11]$ for more detail. Here, we propose an alternative (diffuserbased) method for reducing the twin image and one of the dc terms. There are some disadvantages of this approach compared with traditional PSI techniques: more captures are necessary $(>15)$, and recovery of the quantative phase is not straightforward (and is not pursued here); however, other features of the $\mathrm{DH}$ recording process are preserved, namely, we can reconstruct our object at different depths and from different perspectives. We note that this approach also reduces speckle noise in our reconstructions. In the following section we present a simple (1D) theoretical model to describe the behavior of our optical system. Our aim here is not to conduct a fully rigorous examination of the complex interaction of multiple speckle fields and various apertures in the system but rather to present a plausible description of the system behavior. A collimated plane wave was generated by using a spatial filter and lens. This collimated plane wave is then incident on a diffuser. We assume that the diffuser is optically rough and imparts a random phase to the plane wave front that emerges from the diffuser. This random phase field now propagates to the object plane, where it illuminates our transmissive target. We note that the random phase field at the output of the diffuser gives rise to both random amplitude and phase values at our object plane, owing to diffraction introduced by the finite extent of the diffuser $[12,13]$. Thus we describe the random field that illuminates our object as

$$
u_{\mathrm{R}}(X)=a_{\mathrm{R}}(X) \exp \left[j \phi_{\mathrm{R}}(X)\right],
$$

where $a_{\mathrm{R}}(X)$ and $\phi_{\mathrm{R}}(X)$ are random amplitude and phase values, respectively, and where $j=\sqrt{-1}$. We use the variable $X$ to refer to the coordinate space in the object and reconstruction planes and the variable $x$ to refer to the coordinate space in the hologram plane. We describe the effect of our transmissive object as

$$
u_{\mathrm{OB}}(X)=a_{\mathrm{OB}}(X) \exp \left[j \phi_{\mathrm{OB}}(X)\right]
$$

and write the field immediately after our object as

$$
u(X)=u_{\mathrm{OB}}(X) u_{\mathrm{R}}(X) .
$$

This combined field, $u(X)$, propagates to the hologram (CCD) plane and interferes with an ideal unit amplitude plane wave, $\operatorname{ref}(x)=\exp [j \Omega]$ (where $\Omega$ is some arbitrary phase), and the resulting interference pattern is recorded. The continuous intensity distribution incident upon the camera plane can be expressed as

$$
\begin{aligned}
H(x)=\left|u_{z}(x)+\operatorname{ref}(x)\right|^{2}= & I_{z}+I_{\text {ref }}+u_{z}(x) \operatorname{ref}^{*}(x) \\
& +u_{z}^{*}(x) \operatorname{ref}(x),
\end{aligned}
$$

where $I_{z}$, and $I_{\text {ref }}$ are the dc terms corresponding to the object and reference intensities, respectively, and * denotes the complex conjugate operation. The latter two terms in Eq. (4) correspond to the real and twin image terms, respectively. The field $u_{z}$ is related to our object field $u(X)$ by a Fresnel transform [14,15]: 


$$
u_{z}(x)=\mathfrak{I}_{z}\{u(X)\}(x)=\frac{1}{\sqrt{j \lambda z}} \int u(X) \exp \left[\frac{j \pi}{\lambda z}(x-X)^{2}\right] \mathrm{d} X,
$$

where $\mathfrak{I}_{z}\{\}$ is the Fresnel transform operator and $\lambda$ is the wavelength of the light. We now make some more simplifying approximations. In practical DH systems the continuous intensity field $H(x)$ is recorded by a camera of finite physical extent using finite size pixels located at fixed distances from each other. Each of these factors limits the imaging performance of $\mathrm{DH}$ systems [15]. However, for our purposes, we do not need to consider these aspects of the imaging system to get across the essence of our idea. For simplicity we assume that the continuous intensity distribution $H(x)$, of Eq. (4), is available to us. We can remove the dc terms by recording the reference and object intensities separately and subtracting them numerically from the captured hologram. Alternatively, numerical techniques can be used to remove these terms [16]. For example, below we use a simple Fourier filtering operation to remove $I_{\text {ref }}$. We also demonstrate experimentally that the $I_{z}$ term is removed by using our technique. Recalling that $\operatorname{ref}(x)=\exp [j \Omega]$ and, choosing $\Omega=0$, in Eq. (4) we remove the dc terms and perform an inverse Fresnel transform, using a computer to numerically reconstruct the hologram. This yields the following result:

$$
g(X)=u(X)+\mathfrak{I}_{-z}\left\{u_{z}^{*}(x)\right\}(X)=u(X)+\widetilde{u}(X),
$$

where $\widetilde{u}(X)$ is the twin image term. The intensity of this numerical reconstruction is given by

$$
\begin{aligned}
I^{\mathrm{recon}}=g(X) g^{*}(X)= & u(X) u^{*}(X)+\tilde{u}(X) \tilde{u}^{*}(X)+|u(X)| \\
& \times|\widetilde{u}(X)| \cos \left(\vartheta_{R}\right), \\
I^{\text {recon }}= & I^{\text {real }}+I^{\text {twin }}+\mathrm{CT},
\end{aligned}
$$

where $\vartheta_{R}$ is a random variable and CT refers to the cross terms in Eq. (7a). Our twin removal method requires that we capture multiple digital holograms, using a series of statistically independent speckle fields to illuminate our object. From here on we use the index $n$ to denote different captures. We assume that each of these statistically independent fields has the same average intensity $M$. Each of the resulting digital holograms, having first had the $I_{\text {ref }}$ dc term removed, are then reconstructed and averaged on an intensity basis in the image plane, giving

$$
I_{\mathrm{AV}}^{\mathrm{recon}}=\frac{1}{N}\left(\sum_{n=1}^{N} I_{n}^{\mathrm{real}}+\sum_{n=1}^{N} I_{n}^{\mathrm{twin}}+\sum_{n=1}^{N} \mathrm{CT}_{n}\right) .
$$

For a given diffuse illumination the resulting real image intensity term can be expressed as [by using Eqs. (1)-(3) and Eq. (7)]

$$
I_{n}^{\text {real }}=\left(\left|a_{\mathrm{OB}}\right|\left|a_{\mathrm{R} n}\right|\right)^{2} .
$$

Averaging $N$ of these intensity distributions (each due to a statistically independent speckle field), we can express the first term of Eq. (8) as

$$
\sum_{n=1}^{N} I_{n}^{\text {real }}=\left|a_{\mathrm{OB}}\right|^{2}\left(\frac{\left|a_{\mathrm{R} 1}\right|^{2}+\ldots+\left|a_{\mathrm{RN}}\right|^{2}}{N}\right)=\left|a_{\mathrm{OB}}\right|^{2}(M),
$$

where $M$ is the sum of $N$ statistically different intensity patterns. As $N$ goes to infinity, we may replace this sum by the average intensity value for a given speckle field, $M$. It is important to note that the intensity distribution for our object field, $\left|a_{\mathrm{OB}}\right|^{2}$, is contained in Eq. (10). We now consider the second term in Eq. (8). From Theorem 3 in [14], we find that $u_{\mathrm{z}}^{*}(x)=\mathfrak{I}_{-z}\left\{u^{*}(X)\right\}(x)$, and thus $\tilde{u}(X)=\mathfrak{I}_{-2 z}\left\{u^{*}(X)\right\}(X)$. Each corresponding twin intensity term, $I_{n}^{\text {twin }}$, is given by

$$
\begin{aligned}
I_{n}^{\mathrm{twin}}= & \left(\int a_{\mathrm{R} n} a_{\mathrm{OB}} \exp \left[j\left(\phi_{\mathrm{OB}}+\phi_{\mathrm{R} n}\right)\right]\right. \\
& \left.\times \exp \left[\frac{j \pi}{2 \lambda z}\left(X-X_{1}\right)^{2}\right] \mathrm{d} X_{1}\right) \\
& \times\left(\int\left(a_{\mathrm{Rn}} a_{\mathrm{OB}}\right)^{*} \exp \left[-j\left(\phi_{\mathrm{OB}}+\phi_{\mathrm{R} n}\right)\right]\right. \\
& \left.\times \exp \left[\frac{-j \pi}{2 \lambda z}\left(X-X_{2}\right)^{2}\right] \mathrm{d} X_{2}\right) .
\end{aligned}
$$

This result indicates that each twin image intensity distribution generates a statistically independent speckle pattern. As in the previous case the $a_{\mathrm{OB}} \exp \left(j \phi_{\mathrm{OB}}\right)$ term remains invariant. However, now each component is multiplied by a random phase and then Fresnel transformed. Thus averaging over $N$ intensity patterns gives the following result:

$$
\frac{1}{N} \sum_{n=1}^{N} I_{n}^{\mathrm{twin}}=M
$$

This last equation suggests that the twin image reduces to a dc value of $M$ as subsequent intensity distributions are averaged together. Finally it is important to consider the cross terms, $\mathrm{CT}_{n}$ (interference between the real and the twin image) that arise when we calculate the intensity of Eq. (6).

Since $\vartheta_{R}$ in Eq. (7) can be shown to be a random variable, we see that the third term in Eq. (8) will average to zero and can be neglected. While the theoretical description provided here is relatively simplistic, it broadly describes the underlying physical behavior of the system. We shall now demonstrate this with a series of experimental results.

A typical in-line (transmissive geometry) digital holographic system was built. The light source was a $785 \mathrm{~nm}$ CrystalLaser, and the camera was an AVT Dolphin F-145B with $1392 \times 1040$ pixels and a pixel pitch of $6.45 \mu \mathrm{m}$. By use of a piezo mirror (PiezoSystemJena PZ38CAP with controller NV40/1CLE) and numerical phase-shifting techniques, a twin and dcfree PSI hologram was captured for comparative purposes. A diffuser was then mounted on a translation stage and introduced into the object arm of the sys- 
tem to generate the speckle fields used to illuminate the object. By moving the position of the diffuser between holographic captures (by a distance of approximately $5 \mathrm{~mm}$ ), a series of statistically independent speckle patterns was generated. The reconstruction distance for the holograms was $106.18 \mathrm{~mm}$. They were numerically reconstructed by using the direct method to implement the discrete Fresnel transform [15]. The images presented in Figs. 1(a)-1(f) are numerical reconstructions of the test object-the USAF resolution chart.

Figure 1(a) shows a reconstruction that contains the two dc, the twin, and the object terms (we note that the brightness of Fig. 1(a) has been lowered to allow all the information to be seen). In Fig. 1(b) the $I_{\text {ref }}$ term has been removed by a numerical high-pass filter. Since the reference field was a plane wave, most of the reference power is mapped to the center of a Fourier transform of the hologram, where it is removed with a Gaussian-shaped high-pass filter (40 $\times 40$ pixels). Here we make no attempt to remove the $I_{z}$ term. However, it can seen from the experimental results that $I_{z}$ is significantly reduced by the averaging process. In Fig. 1(c) the twin image has been reconstructed as a speckle pattern because of the intro-

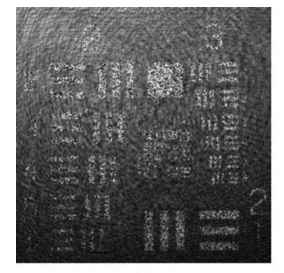

(a)

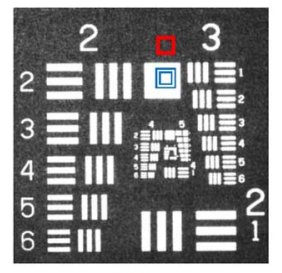

(d)

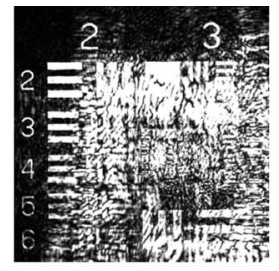

(b)

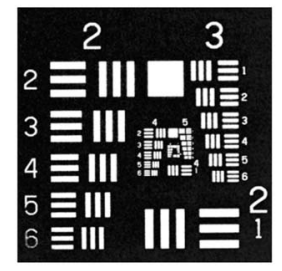

(e)

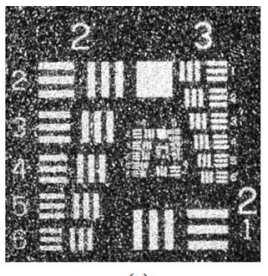

(c)

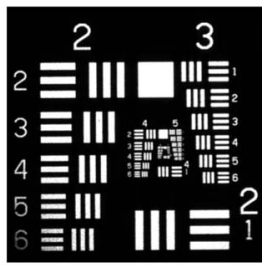

(f)
Fig. 1. (Color online) (a) Reconstruction of the unprocessed original hologram, which contains the two dc, object, and twin terms. (b) Same reconstruction where $I_{\text {ref }}$ has been removed by using a numerical high-pass filter. The twin image appears as corrupting noise in the reconstruction. In (c) a diffuser has been placed in the object beam prior to illuminating the object. The twin is also present in the resulting reconstruction; however, it now appears as a speckle pattern spread out over the entire reconstruction plane. (d) shows the result of averaging together 35 holographic reconstructions, each with a statistically independent speckle pattern. This averaging process reduces the speckle contrast and also the twin image, changing it into a constant dc value. The background dc has been subtracted numerically in (e) and can be compared with a reconstruction from a PSI hologram in (f). duction of a diffuser in the object path. Figure 1(d) shows the results when 35 separate holograms have been averaged on an intensity basis. It can be seen that the twin term has been significantly reduced when compared with Fig. 1(c). These additions produce a background dc term [see Eq. (9)] that can be removed [see Fig. 1(e)] by subtracting the mean value of the background from the entire reconstruction. Figures 1(e) and 1(f) show a comparison between our twin removal method, Fig. 1(e), and a PSI method, Fig. 1(f). The size of the deviation from the PSI hologram [Fig. 1(f)] is related to the reduction in speckle contrast and is given by the formula $\sqrt{1 / N}$ [12]. The background and image speckle contrast reduction was calculated over a $25 \times 25$ pixel area, as indicated by the squares in Fig. 1(d) and was found to agree with this formula. We estimate that there is a normalized maximum error of $20 \%$ between Figs. 1(e) and $1(f)$.

In this Letter we have described a technique for reducing the effect of the twin term and attenuating the $I_{z} \mathrm{dc}$ term in $\mathrm{DH}$. The latter result can be derived theoretically by including the $I_{z}$ term in Eq. (7). Following a similar analysis it can be shown that the $I_{z}$ term is also averaged out. Finally, we believe that the results here also extend to a reflective geometry [17].

We acknowledge funding from the EC's Seventh Framework Programme 2007-2013 under grant agreement 216105 and from Science Foundation Ireland.

\section{References}

1. D. Gabor, Nature 161, 777 (1948).

2. D. Gabor, Proc. R. Soc. London, Ser. A 197, 454 (1949).

3. M. Testorf and A. W. Lohmann, Appl. Opt. 47, A70-A77 (2008).

4. E. N. Leith and J. Upatnieks, J. Opt. Soc. Am. 52, 1123 (1962).

5. J. W. Goodman and R. W. Lawrence, Appl. Phys. Lett. 11, 77 (1967).

6. U. Schnars and W. Jüptner, Appl. Opt. 33, 179 (1994).

7. T. M. Kreis, Opt. Eng. 41, 1829 (2002).

8. A. W. Lohmann, R. G. Dorsch, D. Mendlovic, Z. Zalevsky, and C. Ferreira, J. Opt. Soc. Am. A 13, 470 (1996).

9. I. Yamaguchi and T. Zhang, Opt. Lett. 22, 1268 (1997).

10. G. S. Han and S. W. Kim, Appl. Opt. 33, 7321 (1994).

11. Z. Wang and B. Han, Opt. Lett. 29, 1671 (2004).

12. J. W. Goodman, Speckle Phenomena in Optics (Roberts, 2007).

13. J. W. Goodman, J. Opt. Soc. Am. 66, 1145 (1976).

14. F. Gori, Opt. Commun. 39, 293 (1981).

15. D. P. Kelly, B. M. Hennelly, N. Pandey, T. J. Naughton, W. T. Rhodes, Opt. Eng. 48, 095801 (2009).

16. T. Kreis and W. Juptner, Opt. Eng. 36, 2357 (1997).

17. D. S. Monaghan, D. P. Kelly, N. Pandey, and B. M. Hennelly, Presented at China-Ireland International Conference on Information and Communications Technologies (Maynooth, Ireland, August 19-21, 2009). 